Сапон И. В. ${ }^{1}$

Сибирский государственный университет телекоммуникаций и информатики, ул. Кирова, 86, Новосибирск, 630102, Россия

${ }^{1}$ irina.sapon@bk.ru

2dled04@yandex.ru

Статья поступила 18 января 2020 г.; принята 2 мая 2020 г.; опубликована 30 июня 2020 г.

Аннотация. В цифровой век изменились процессы, связанные с человеческим общением и приватностью. Дружба в социальной сети не требует больших эмоциональных и временных затрат, а онлайн-демонстрация себя и чрезмерное раскрытие личных данных стали трендом современности. В статье рассматривается вопрос о том, как возросшее количество социальных контактов связано с самораскрытием в профиле социальной сети. Был проведён контент-анализ личных страниц 1707 пользователей «ВКонтакте», который показал, что число друзей оказывает определенное влияние на тип и объём публикуемой информации. Так, пользователи с бо́льшим количеством сетевых друзей в целом чаще раскрывают личную информацию в профиле. Данный вывод согласуется с результатами зарубежных исследований, что позволяет предположить, что обнаруженный механизм является универсальным, так как встречается в различных культурных контекстах. Также в нашей работе было показано, что количество «друзей» положительно связано с раскрытием таких типов информации, как: «Деятельность», «Интересы», «Статус», «Номер телефона», «Ссылки на аккаунты в других социальных сетях». Авторы приходят к выводу, что данная связь может обуславливаться рекламными мотивами. В частности, раскрытие контактной информации создаёт возможность быстрой связи с клиентами, а заполнение полей «Деятельность» и «Статус» помогает в презентации услуг. Данная работа вносит вклад в понимание факторов самораскрытия в профиле и предлагает один из вариантов объяснения «парадокса приватности».

Ключевые слова: самораскрытие; социальные сети; профиль; личная информация; приватность; сетевые друзья; «ВКонтакте»

Благодарность. Исследование выполнено при финансовой поддержке РФФИ и Правительства Новосибирской области в рамках научного проекта № 19-411543002 «Исследование самораскрытия в профиле на примере социальной сети «ВКонтакте».

Информация для щитирования: Сапон И. В., Леденев Д. Е. Виртуальная личность: сетевой эффект самораскрытия // Научный результат. Социология и управление. 2020. Т. 6, № 2. С. 36-50. DOI: 10.18413/2408-9338-2020-6-2-0-3 


\title{
Virtual Identity: The Network Effect of Self-Disclosure
}

Siberian State University of Telecommunications and Informatics

86 Kirov St., Novosibirsk, 630102, Russia

1irina.sapon@bk.ru

2dled04@yandex.ru

\section{Received on January 18, 2020; accepted on May 02, 2020;} published on June 30, 2020

\begin{abstract}
In the digital age, communication and privacy processes have changed. Friendship maintenance in SNS does not require a lot of emotional and time expenses, while self-presentation and self-disclosure have become a modern trend. The article discusses the relationship between the number of network friends and self-disclosure in the social network site (SNS) profile. The content analysis of 1707 VKontakte user's personal pages was conducted, which showed that the number of friends affects the type and amount of published information. For example, users with a large number of online friends are generally more likely to disclose personal data in their profile. This is especially true for the profile fields such as: «Activities», «Interests», «Status», «Phone Number», «Links to accounts in other SNS». The authors suggest that these results can be explained by the user's advertising motives. In particular, contact information disclosure makes it possible to quickly communicate not only with friends, but also with customers. At the same time, «Activities» and «Status» disclosure helps to promote goods and services. This work contributes to the understanding of self- realization factors in the SNS profile and offers one of the ways to explain the "privacy paradox». Future research should also look at other factors that may affect the self-disclosure in the SNS profile.

Keywords: self-disclosure; social network sites; profile; personal information; privacy; network friends; VKontakte.

Acknowledgements. The reported study was funded by RFBR and Novosibirsk region according to the research project № 19-411-543002 «Research of self-disclosure in the profile of social network site «VKontakte».

Information for citation: Sapon, I. V. and Ledenev, D. E. (2020), "Virtual Identity: The Network Effect of Self-Disclosure", Research Result. Sociology and management, 6 (2), 36-50, DOI: 10.18413/2408-9338-2020-6-2-0-3
\end{abstract}

Введение (Introduction). Социальные сети стали популярным средством коммуникации в современном мире. Они позволяют людям оставаться на связи со своими друзьями и близкими независимо от расстояния: чтобы поделиться какими-либо новостями или событиями достаточно сделать всего пару «кликов» (Stone, 2008). Для многих пользователей ежедневное обновление статуса, а также публикация личных фотографий и видеоисторий («stories») стали привычным действием. При этом они могут не замечать, что публикуют намного больше информации о себе, чем планировали до регистрации, и не задумываются о том, что подробности их личной жизни становятся доступными даже малознакомым людям (Walther, 2007; Hollenbaugh \& Ferris, 2014).

В эпоху социальных сетей демонстрация себя в онлайн-пространстве стала особенно популярной - мы переживаем «революцию социальных данных» (Чернышов, 2017). В то же время личная информация пользователей приобретает определённую 
ценность: её собирают и анализируют коммерческие компании для предложения микротаргетинговой рекламы. Она может использоваться в преступных целях, например, фотографии из отпуска помогают преступникам в планировании времени ограбления. И если предложение рекламы не представляет для пользователя какую-либо серьезную опасность, то риск попадания личной информации в руки к злоумышленникам может серьёзно навредить пользователю.

Логично предположить, что пользователи будут стремиться защитить конфиденциальность своих данных, скрывая их настройками приватности или же удаляя со страницы социальной сети. Однако, как показали результаты исследований, пользователи не предпринимают необходимых мер по защите своей приватности, даже если осознают потенциальные риски от раскрытия личной информации в онлайн-пространстве. Такое нелогичное поведение получило название «парадокс приватности» (Kokolakis, 2017). Почему пользователи продолжают публиковать личную информацию несмотря на потенциальную опасность? Что влияет на их решение?

Ответы на эти вопросы помогут лучше понять поведение пользователей в онлайн-пространстве, что в целом будет способствовать созданию безопасной и комфортной интернет-среды. Также это поможет привлечь внимание к проблеме приватности персональных данных, в результате чего решение пользователей о публикации личной информации в сети может стать более осознанным.

Данная работа является частью более масштабного исследования, посвящённого изучению факторов самораскрытия в профиле социальной сети. В качестве теоретической основы мы используем теорию управления приватностью Сандры Петронио (ТУП). В предыдущей работе мы показали, что данная теория даёт достаточно широкое представление о факторах, влияющих на решение пользователя о саморас- крытии, таких как культура, гендер, мотивы, контекст, соотношение выгод и рисков (Сапон, Леденев, 2018). Однако ТУП не рассматривает ряд специфичных факторов, которые приобретают значение в онлайнконтексте. Результаты нашего исследования могут внести вклад в расширение данной теории для интернет-среды.

Методология и методы (Methodology and methods). По мнению исследователей, одним из возможных объяснений парадокса приватности можно считать убеждённость пользователей в том, что «всё плохое» происходит не с ними. То есть люди недооценивают риски потери приватности, думая, что негативные события касаются чаще других. Это получило название «оптимистическая предвзятость» (Krasnova, 2009).

Но достаточно ли этого, чтобы объяснить парадокс приватности? При попытке объяснить данный парадокс невозможно обойтись без рассмотрения такого явления, как самораскрытие - намеренной передачи личной информации о себе другим (Masur, 2019). В социальных сетях самораскрытие может происходить в личных сообщениях, в профиле, а также посредством размещения записей или комментариев на стене. В профиле содержится намного больше персональных данных человека, чем в какойлибо другой части социальной сети. Он представляет собой некое досье, в котором пользователи могут указывать как чувствительные данные (например, домашний адрес и религиозные взгляды), так и менее серьезные сведения о себе (например, предпочтения в еде или музыке). В нём также присутствует возможность добавлять личные фото, аудио и видеозаписи.

Самораскрытие в онлайн-пространстве является сложным явлением, на которое могут оказывать влияние множество факторов. Например, возраст и гендер ( $\mathrm{Li}$, Lin, Wang, 2015; Hollenbaugh, 2015), культура (Trepte, Masur 2016; Ardi, Maison, 2014), а также мотивы пребывания пользователя в социальной сети (Febriawan, 2017; 
Crabtree, Pillow, 2018). По мнению некоторых авторов, именно мотивы можно считать ключевым фактором самораскрытия в социальной сети. При этом отмечается, что наибольшее влияние оказывают социальные мотивы, например, желание оставаться на связи с друзьями и знакомыми (Kim, Chung, Ahn, 2014). Но как объяснить это влияние?

Как показали результаты исследований, наличие информации на личной странице может снизить социальные барьеры, возникающие при знакомстве, и способствовать созданию, укреплению и накоплению социальных связей. Например, указанная в профиле информация позволяет сформировать первое впечатление о человеке уже до начала общения, что влияет на решение, подтвердить или же проигнорировать запрос на дружбу (Lampe, 2007; Schrammel, 2009; Bazarova, Choi, 2014).

Таким образом, можно предположить, что пользователи, раскрывающие значительное количество информации на страницах социальной сети, будут иметь и бо́льшее число друзей. И действительно, многие зарубежные исследователи, изучавшие самораскрытие в профиле Facebook, подтвердили это предположение (Kisilevich, 2012; Ahmed, 2015; Aharony, 2016).

Однако стоит заметить, что чем более публичным становится человек, тем больше у него возникает причин быть осторожным в раскрытии, так как в «дружескую» сеть могут попасть злоумышленники, готовые использовать полученную информацию в корыстных целях (Wang, Burke, Kraut, 2016). Поэтому, на наш взгляд, нельзя однозначно сказать, как будет меняться самораскрытие с увеличением количества сетевых друзей. В отечественной социологии нами не были обнаружены исследования по данной теме. Наша работа направлена на то, чтобы заполнить этот пробел и рассмотреть, как данная связь проявляется в контексте российской социальной сети.
Целью данной работы является изучение взаимосвязи между количеством сетевых друзей и самораскрытием в профиле «ВКонтакте». Особенность нашего исследования заключается в том, что мы рассматриваем не только общий объём публикуемых данных, но и определяем, какие типы информации свойственно раскрывать пользователям с бо́льшим количеством друзей. Также мы проводим семантический анализ некоторых типов информации в профиле, чтобы дать более точную и объективную интерпретацию полученных результатов.

Сформулируем гипотезы исследования:

1. Количество сетевых друзей положительно связано с общим объёмом раскрываемой в профиле информации.

2. Пользователи с бо́льшим количеством сетевых друзей чаще раскрывают определенные типы информации.

3. Причины раскрытия информации в профиле, как и причины большого количества сетевых друзей - социальные.

В июле 2019 года мы провели контент-анализ профилей социальной сети «ВКонтакте». Данная платформа была выбрана нами ввиду того, что достаточно долгое время оставалась самой популярной в России социальной сетью. На данный момент она продолжает занимать лидирующую позицию несмотря на возрастающую популярность Instagram (Курносова, 2019).

Контент-анализ включил личную информацию 1707 профилей пользователей, которые являлись сетевыми друзьями одного из авторов и были так или иначе знакомы с ним, что исключило попадание в выборку «фейковых» страниц и «ботов». В одной из наших работ было показано, что $63 \%$ пользователей применяют настройки приватности, скрывая часть данных профиля от тех, кто не входит в список друзей (Сапон, Леденев, 2018). Поэтому для того, чтобы получить доступ к наибольшему количеству информации в профиле необходимо проводить контент-анализ страниц из позиции сетевого друга. Таким образом, несмотря на то, что такой подход исключает 
фактор случайности и несколько смещает выборочную совокупность, он даёт явные преимущества.

Средний возраст пользователей в выборке составил 31,75 лет $(\mathrm{SD}=7,41)$, мужчин было несколько больше, чем женщин (882 против 825 соответственно).
Для изучения самораскрытия в данной сети нами была разработана автоматизированная система сбора и обработки данных.

На первом этапе исследования мы вычислили частоту раскрытия 27 типов информации в профиле (рис. 1). Учитывалось только наличие (1) или отсутствие (0) данных в соответствующем поле личной страницы.

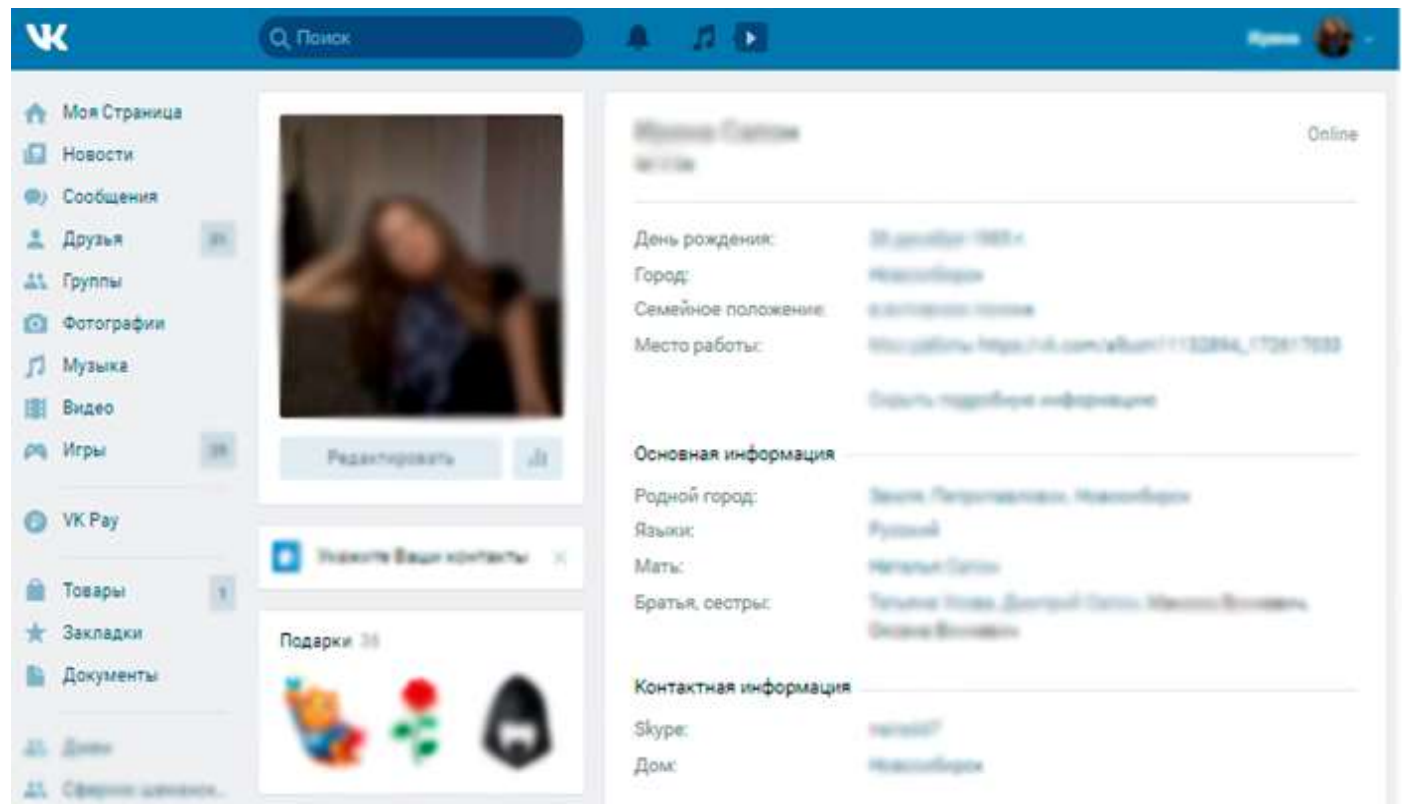

Рис. 1. Профиль в социальной сети «ВКонтакте»

Fig. 1. Profile in the social network site VKontakte

Категория «Аватар» была рассмотрена нами дополнительно с целью исключить из подсчета те фото, которые не содержали личного изображения пользователя.

Раскрытием такого типа информации, как «Номер телефона» мы считали лишь те данные, которые соответствовали определенному формату. Так, номер мобильного телефона должен был содержать 10 или 11 цифр (если он начинается с «+7» или «8»), а городского - 7 цифр.
На втором этапе исследования мы провели семантический анализ по методу А. Браймана (Bryman, 2016) некоторых полей профиля с целью определить тематику раскрываемой информации.

Научные результаты и дискуссия (Research Results and Discussion). Прежде чем проверить основные гипотезы нашего исследования, мы рассмотрим, сколько сетевых друзей у пользователей в нашей выборке. Эти данные представлены на гистограмме ниже (рис. 2). 


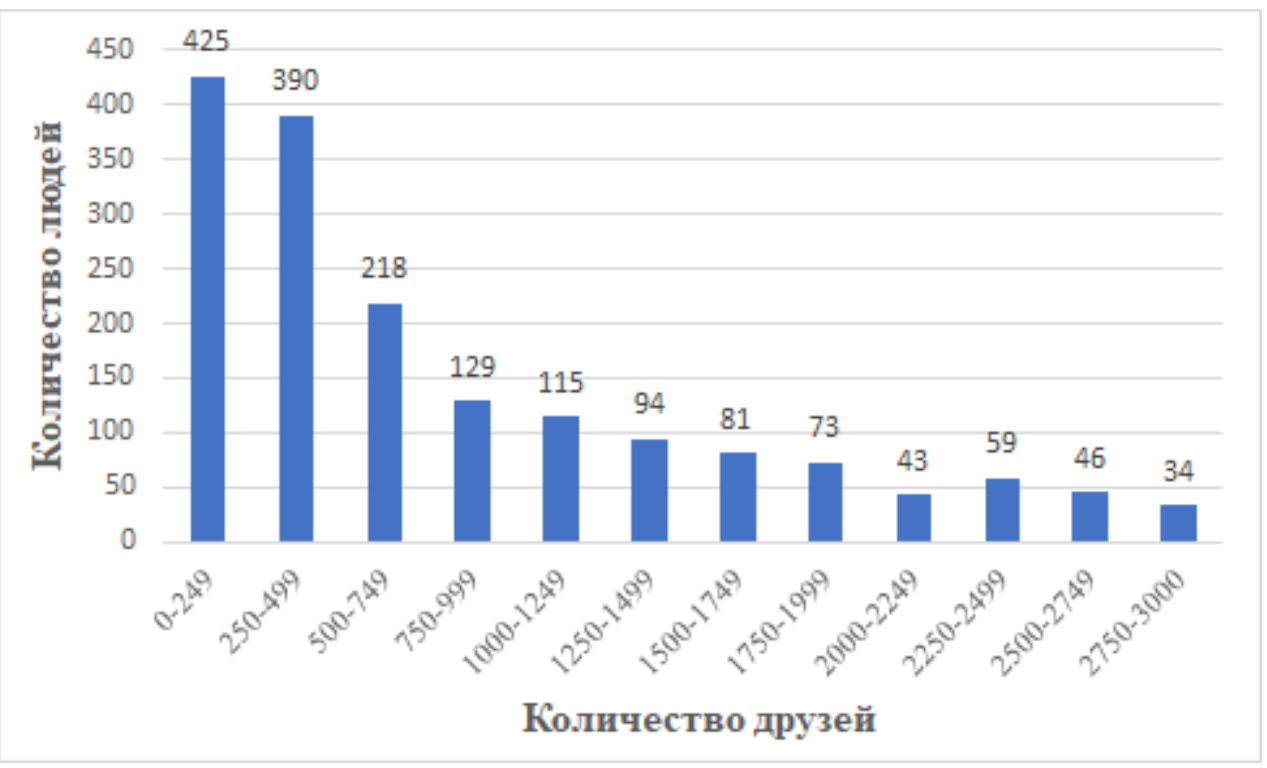

Рис. 2. Количество «друзей» у пользователей в выборке

Fig. 2. Number of users" «friends» in the sample

Как видим, только у 425 пользователей $(24,8 \%$ выборки) в списке друзей находится до 250 человек. У других же пользователей сетевых друзей гораздо больше. В целом среднее количество составляет 830,28 (SD=756,56). Это значение превышает «число Данбара» (150) - предельное количество социальных связей, которые способен поддерживать человек (Roberts, Dunbar, Pollet, Kuppens, 2009).

Но чем можно объяснить такое значительное количество «дружеских» связей? Дело в том, что социальные сети изменили понятие «друг», упростив процесс знакомства. Такая «дружба» не требует больших вложений и не предполагает сильной эмоциональной вовлечённости. Это позволяет добавлять в список друзей даже малознакомых людей. Список друзей в социальной сети - это онлайн-коллекция социальных связей человека, нечто вроде телефонной книги.

С точки зрения концепции социального капитала, люди получают определён- ные преимущества от большого количества социальных связей. Это увеличивает потенциальную возможность получения эмоциональной поддержки, какой-либо информации или же иных ресурсов (Stefanone, Kwon, Lackaff, 2011). К тому же обширный список сетевых друзей является признаком популярности и привлекательности. Поэтому пользователи нередко добавляют незнакомцев. Соответственно, самораскрытие в профиле социальной сети происходит не только для близких, но и для малознакомой аудитории.

Но действительно ли с расширением аудитории увеличивается объём самораскрытия в профиле?

Проведённый корреляционный анализ Пирсона показал, что между общим уровнем самораскрытия в профиле и числом сетевых друзей существует связь и она положительная $(\mathrm{r}=0,14, \mathrm{p}<0,05)$. Графически данная зависимость показана на рис. 3. 


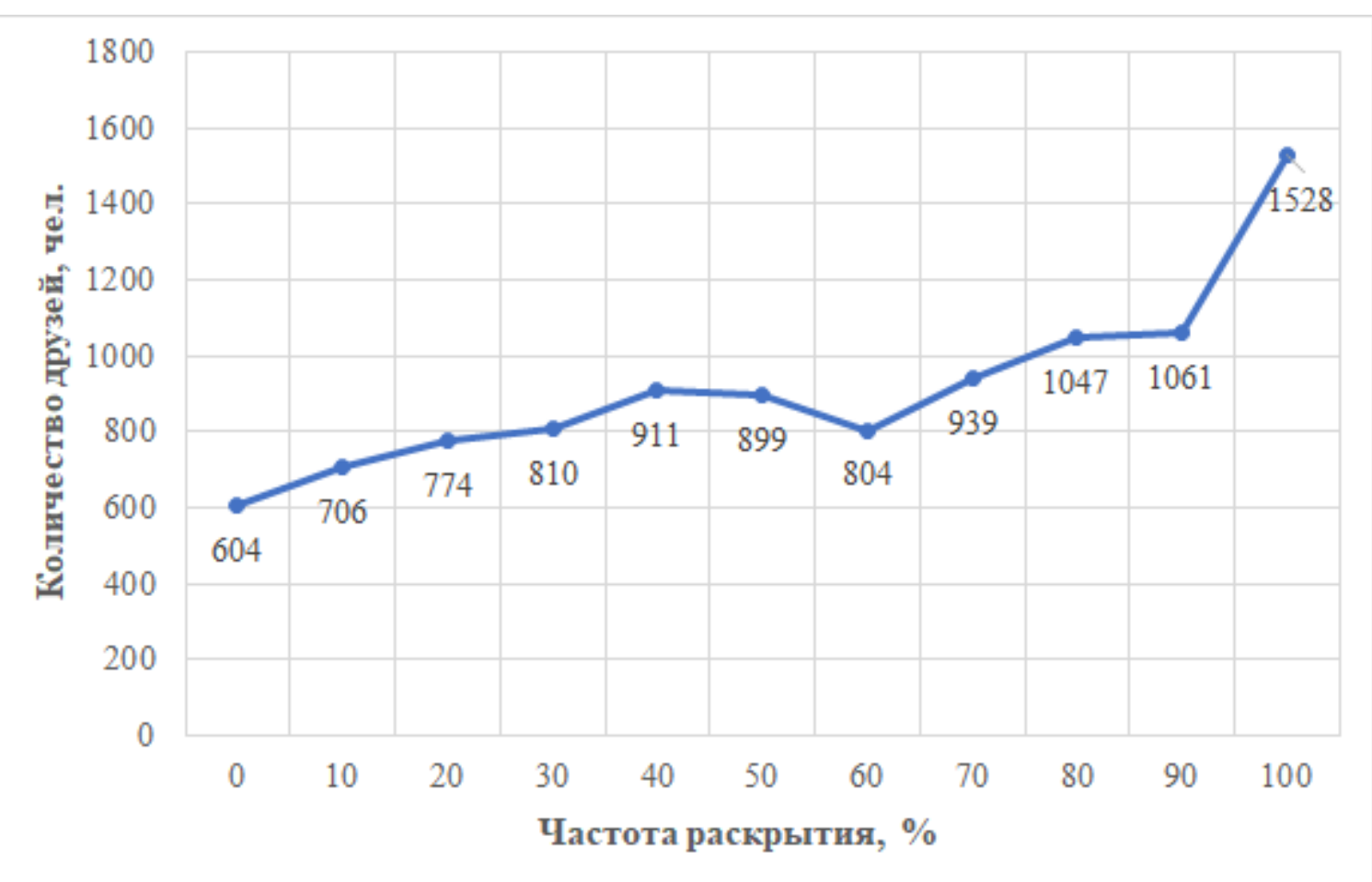

Рис. 3. Зависимость между общим уровнем самораскрытия в профиле и числом дружеских связей

Fig. 3. Relationship between the self-disclosure level in the profile and the number of network friends

Как видим, чем больше у пользователей сетевых друзей, тем в среднем чаще они раскрывают информацию в профиле. Этот вывод согласуется с результатами зарубежных исследований, в которых также была замечена связь онлайн-самораскрытия и количества друзей в социальной сети Facebook (Lee, 2012; Kim, Lee, 2011). Bepoятно, данный механизм является универсальным, так как встречается в разных культурных контекстах. Различная информация в профиле может действовать как социальные сигналы, которые могут рассказать что-либо о пользователе. Это снижает время на поиск общих тем и интересов, улучшает понимание между участниками сети (Schrammel, 2009). Возможно, заполненный профиль действительно способствует накоплению социальных связей.

Но какие типы информации пользователи с бо́льшим количеством социальных связей раскрывают чаще остальных? Для дальнейшего анализа мы разделили пользователей нашей выборки на четыре группы в зависимости от количества их сетевых друзей. Такое разбиение позволило нам получить примерно равное число людей в каждой из групп (табл. 1).

Таблица 1

Группировка пользователей в зависимости от количества сетевых друзей

Table 1

Grouping users based on the number of network friends

\begin{tabular}{|l|c|c|c|c|}
\hline \multicolumn{1}{|c|}{ Количество сетевых друзей } & До 250 & От 250 до 500 & $\begin{array}{c}\text { От 500 } \\
\text { до 1250 }\end{array}$ & $\begin{array}{c}\text { От } 1250 \text { до } \\
3000\end{array}$ \\
\hline Раскрытие в профиле, \% & 31,98 & 39,60 & 40,01 & 42,53 \\
\hline Количество людей, чел. & 425 & 390 & 462 & 430 \\
\hline
\end{tabular}


С помощью критерия хи-квадрат мы сравнили данные группы по частоте раскрытия 27 типов информации. Все раскрываемые в профиле данные мы также разделили на четыре категории: «Основная информация»,

«Мировоззрение», «Интересы», «Медиаактивность». Приведём полученные результаты.

Категория «Основная информация» включила стандартные сведения о пользователе: личное фото (аватар), статус, возраст, контактные данные (табл. 2).

Таблица 2

Частота раскрытия категории «Основная информация», \%

Table 2

Disclosure frequency of the «Basic information» category», \%

\begin{tabular}{|l|c|c|c|c|c|}
\hline $\begin{array}{c}\text { Количество друзей / } \\
\text { Тип информации }\end{array}$ & до 250 & $\begin{array}{c}\text { от 250 } \\
\text { до 500 }\end{array}$ & $\begin{array}{c}\text { от } 500 \text { до } \\
1250\end{array}$ & $\begin{array}{c}\text { от } 1250 \\
\text { до 3000 }\end{array}$ & $\begin{array}{c}\text { Значение } \\
\text { хи-квадрат }\end{array}$ \\
\hline Аватар & 73,65 & 76,98 & 76,36 & 75,12 & 0,09 \\
\hline Статус & 42,82 & 54,73 & 63,34 & 74,65 & $9,23^{*}$ \\
\hline Семейное положение & 33,41 & 37,08 & 39,05 & 38,84 & 0,55 \\
\hline Возраст & 32 & 38,46 & 33,12 & 30,7 & 1,04 \\
\hline Город & 81,41 & 85,93 & 83,08 & 87,67 & 0,28 \\
\hline $\begin{array}{l}\text { Ссылки на аккаунты в других } \\
\text { социальных сетях }\end{array}$ & 16,94 & 31,71 & 34,92 & 38,6 & $8,86^{*}$ \\
\hline Телефон & 19,76 & 33,25 & 39,05 & 43,02 & $9,18^{*}$ \\
\hline Образование & 61,18 & 71,61 & 69,2 & 68,6 & 0,9 \\
\hline
\end{tabular}

* - статистически значимая разница $(\mathrm{p}<0.05)$

** - статистически значимая разница $(\mathrm{p}<0.01)$

Как можно заметить, пользователи с бо́льшим количеством сетевых друзей в среднем чаще публикуют контактную информацию («Телефон», «Ссылки на аккаунты в других социальных сетях») и заполняют поле «Статус». Таким образом, пользователи, которые сообщают о том, как с ними можно связаться, более открыты к социальным контактам и сознательно расширяют список друзей в социальной сети.
Перейдём к рассмотрению следующей категории, которая содержит сведения о предпочтениях пользователя. Статистически значимая разница была обнаружена при сравнении частоты заполнения таких полей профиля, как «Деятельность» и «Интересы». С увеличением количества сетевых друзей увеличивалось и раскрытие данных типов информации (табл. 3).

Частота раскрытия категории «Интересы», \%

Таблица 3

Table 3

Disclosure frequency of the «Interests» category, \%

\begin{tabular}{|l|c|c|c|c|c|}
\hline $\begin{array}{c}\text { Количество друзей / } \\
\text { Тип информации }\end{array}$ & до 250 & $\begin{array}{c}\text { от } 250 \\
\text { до 500 }\end{array}$ & $\begin{array}{c}\text { от } 500 \text { до } \\
1250\end{array}$ & $\begin{array}{c}\text { от } 1250 \\
\text { до 3000 }\end{array}$ & $\begin{array}{c}\text { Значение } \\
\text { хи-квадрат }\end{array}$ \\
\hline Деятельность & 16,47 & 29,92 & 44,47 & 50,47 & $19,74 * * *$ \\
\hline Интересы & 21,18 & 34,02 & 39,05 & 43,95 & $8,33 *$ \\
\hline Любимая музыка & 21,65 & 27,37 & 30,15 & 32,09 & 2,23 \\
\hline Любимые фильмы & 19,53 & 27,62 & 25,6 & 26,98 & 1,65 \\
\hline Любимые книги & 17,65 & 23,53 & 26,25 & 27,21 & 2,34 \\
\hline Любимые игры & 12,94 & 20,72 & 21,48 & 19,3 & 2,43 \\
\hline Любимые цитаты & 23,53 & 34,78 & 34,49 & 35,58 & 3,07 \\
\hline О себе & 17,65 & 27,62 & 34,49 & 36,98 & 7,69 \\
\hline
\end{tabular}

* - статистически значимая разница $(\mathrm{p}<0.05)$

** - статистически значимая разница $(\mathrm{p}<0.01)$ 
Возможно, раскрывая информацию о своих увлечениях и роде деятельности, люди пытаются расширить круг своих единомышленников.

Далее рассмотрим категорию «Жизненная позиция», которая включает в себя сведения о жизненных установках пользователя, например, его отношение к курению или к алкоголю, а также политические, религиозные и иные взгляды. Следует отметить, что значительной разницы в частоте раскрытия данных типов информации обнаружено не было (табл. 4).

Таблица 4

Частота раскрытия категории «Жизненная позиция», \%

Table 4

Disclosure frequency of the «Life position» category», \%

\begin{tabular}{|l|c|c|c|c|c|}
\hline $\begin{array}{c}\text { Количество друзей / } \\
\text { Тип информации }\end{array}$ & до 250 & $\begin{array}{c}\text { от } 250 \\
\text { до } 500\end{array}$ & $\begin{array}{c}\text { от } 500 \text { до } \\
1250\end{array}$ & $\begin{array}{c}\text { от } 1250 \\
\text { до } 3000\end{array}$ & $\begin{array}{c}\text { Значение } \\
\text { хи-квадрат }\end{array}$ \\
\hline Политические предпочтения & 19,06 & 21,74 & 20,39 & 20,23 & 0,18 \\
\hline Мировоззрение & 22,35 & 30,43 & 29,28 & 30,7 & 1,65 \\
\hline Главное в жизни & 18,82 & 24,81 & 21,69 & 29,53 & 2,66 \\
\hline Главное в людях & 19,53 & 23,79 & 21,26 & 26,05 & 1,08 \\
\hline Отношение к курению & 19,53 & 23,79 & 20,61 & 22,56 & 0,51 \\
\hline Отношение к алкоголю & 20 & 23,27 & 20,17 & 22,56 & 0,38 \\
\hline Источники вдохновения & 12,24 & 18,41 & 19,74 & 24,19 & 3,92 \\
\hline
\end{tabular}

Теперь рассмотрим категорию «Медиаактивность». Она отражает, к какому личному контенту, накопленному в процессе пребывания в социальной сети, пользователь предоставил доступ. Это могут быть списки групп, аудиозаписей или

подарков, а также фотографии, на которых владелец профиля был отмечен другими. При сравнении частоты раскрытия данных типов информации между группами пользователей существенной разницы также обнаружено не было (табл. 5).

Таблица 5

Table 5

Disclosure frequency of the «Media activity» category, \%

\begin{tabular}{|l|c|c|c|c|c|}
\hline $\begin{array}{c}\text { Количество друзей / } \\
\text { Тип информации }\end{array}$ & до 250 & $\begin{array}{c}\text { от } 250 \\
\text { до } 500\end{array}$ & $\begin{array}{c}\text { от } 500 \text { до } \\
1250\end{array}$ & $\begin{array}{c}\text { от } 1250 \\
\text { до } 3000\end{array}$ & $\begin{array}{c}\text { 3начение } \\
\text { хи-квадрат }\end{array}$ \\
\hline Фото с пользователем & 40 & 54,48 & 52,93 & 50,47 & 2,58 \\
\hline Список аудиозаписей & 71,29 & 77,75 & 73,32 & 73,26 & 0,3 \\
\hline Список групп & 55,29 & 53,2 & 50,33 & 50,47 & 0,33 \\
\hline Список подарков & 53,65 & 61,89 & 56,83 & 68,6 & 2,12 \\
\hline
\end{tabular}

Подытожим сказанное. Как было показано выше, статистически значимая разница наблюдалась в частоте указания следующих типов информации: «Деятельность», «Интересы», «Статус», «Номер телефона», «Ссылки на аккаунты в других социальных сетях». При этом наибольшая разница была обнаружена при сравнении частоты раскрытия поля «Деятельность». Стоит отметить, что между частотой указания данных типов информации и количеством сетевых друзей наблюдается линейная зависимость. Графически данная связь показана ниже (рис. 4). 


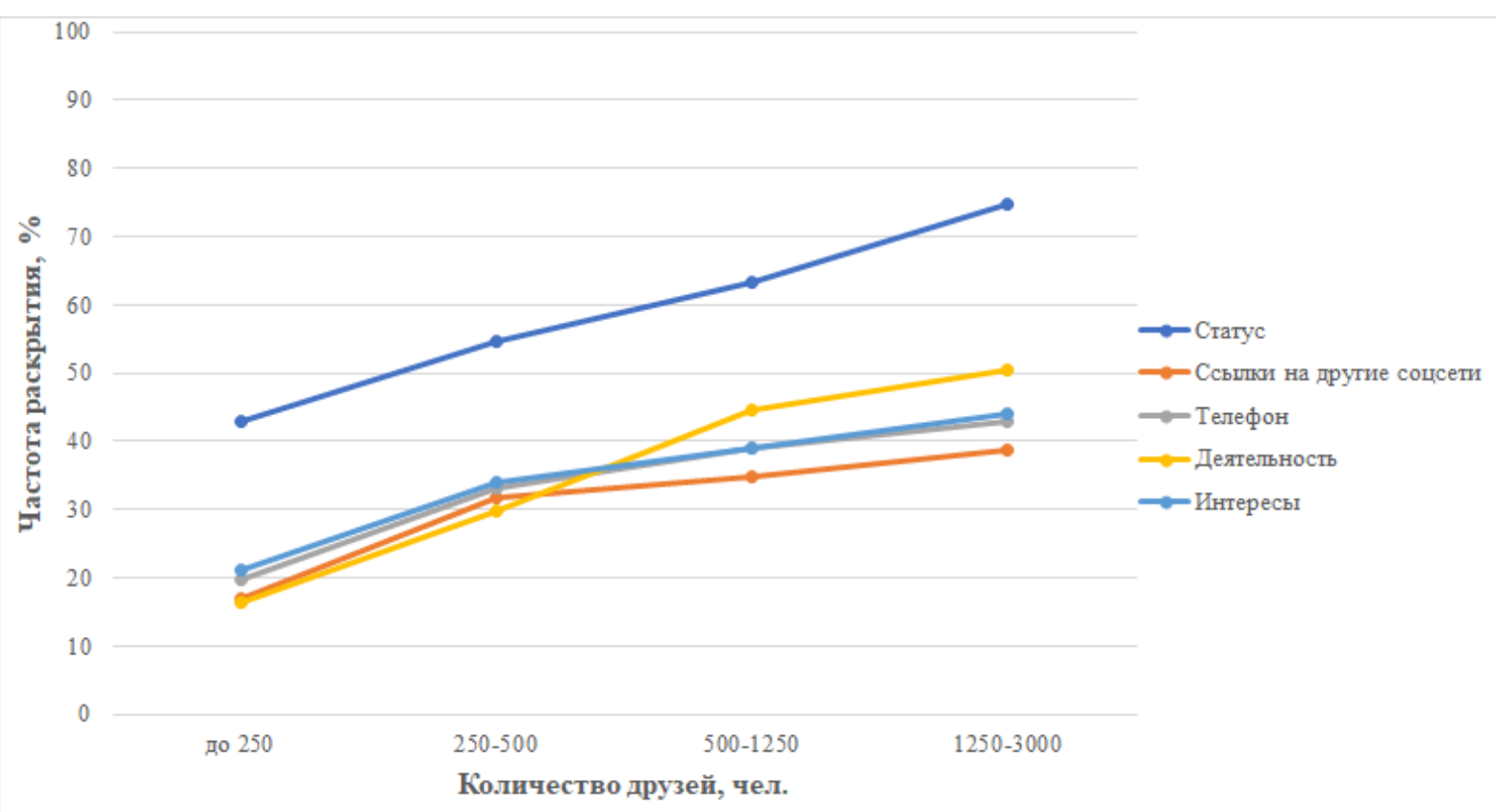

Рис. 4. Взаимосвязь между количеством друзей и раскрытием информации в профиле Fig. 4. Relationship between the number of friends and profile disclosure

Чтобы изучить природу выявленной взаимосвязи мы решили рассмотреть содержание информации в данных полях профиля. Нужно оговориться, что все эти поля заполняются пользователем вручную: при их заполнении не предоставляется возможность выбрать готовый вариант из списка. При этом содержание таких типов информации, как «Номер телефона» и «Ссылки на аккаунты в других социальных сетях» достаточно очевидно и не требует детального рассмотрения. Однако содержание полей «Деятельность», «Интересы» и «Статус», по нашему мнению, заслуживает особого внимания, так как они могут вмещать относительно большой объём текстовой информации разной направленности. Используя методику А. Браймана (Bryman, 2016), мы проанализировали значения данных полей (табл. 6).

\section{Результаты семантического контент-анализа полей «Деятельность», «Интересы» и «Статус», \%}

Таблица 6

Table 6

Results of semantic content analysis of the fields «Activity», «Interests» and «Status», \%

\begin{tabular}{|l|c|c|c|c|c|}
\hline \multicolumn{1}{|c|}{$\begin{array}{c}\text { Количество друзей / } \\
\text { Тип информации }\end{array}$} & $\begin{array}{c}\text { до } \\
250\end{array}$ & $\begin{array}{c}\text { от } 250 \\
\text { до } 500\end{array}$ & $\begin{array}{c}\text { от } 500 \\
\text { до } 1250\end{array}$ & $\begin{array}{c}\text { от } 1250 \\
\text { до } 3000\end{array}$ & $\begin{array}{c}\text { Коэффициент } \\
\text { корреляции } \\
\text { Пирсона }\end{array}$ \\
\hline \multicolumn{5}{|c|}{ Деятельность } \\
\hline $\begin{array}{l}\text { Профессия, род деятельно- } \\
\text { сти }\end{array}$ & 5,2 & 9,5 & 18,2 & 24,0 & $0,142 * * *$ \\
\hline Шутка & 3,5 & 11,3 & 9,5 & 6,7 & 0,034 \\
\hline Реклама & 1,4 & 2,3 & 4,3 & 8,8 & $0,197 * * *$ \\
\hline \multicolumn{7}{|l|}{$\begin{array}{l}\text { Статус } \\
\text { жутка или высказывание о }\end{array}$} & 29,9 & 38,7 & 37,2 & 32,3 & $-0,013$ \\
\hline
\end{tabular}




\begin{tabular}{|l|c|c|c|c|c|}
\hline $\begin{array}{c}\text { Количество друзей / } \\
\text { Тип информации }\end{array}$ & $\begin{array}{c}\text { до } \\
250\end{array}$ & $\begin{array}{c}\text { от } 250 \\
\text { до } 500\end{array}$ & $\begin{array}{c}\text { от } 500 \\
\text { до } 1250\end{array}$ & $\begin{array}{c}\text { от } 1250 \\
\text { до } 3000\end{array}$ & $\begin{array}{c}\text { Коэффициент } \\
\text { корреляции } \\
\text { Пирсона }\end{array}$ \\
\hline $\begin{array}{l}\text { Профессия, род деятельно- } \\
\text { сти }\end{array}$ & 0,5 & 1,5 & 3,7 & 4,9 & $0,296 * * *$ \\
\hline Смайлы, символы & 4,5 & 4,9 & 2,8 & 1,9 & $0,092 * * *$ \\
\hline Местоположение & 0,5 & 1,0 & 1,1 & 0,2 & $-0,070 * *$ \\
\hline Реклама & 7,5 & 8,5 & 18,6 & 35,3 & $-0,022$ \\
\hline Интересы & 8,7 & 15,9 & 18,6 & 23,3 & $0,182 * * *$ \\
\hline Интересы & 3,1 & 2,1 & 8,2 & 9,1 & $0,060 *$ \\
\hline Шутка & 0,2 & 0,5 & 0,6 & 1,4 & 0,001 \\
\hline Реклама
\end{tabular}

*статистически значимая разница $(p<0,05)$

** статистически значимая разница $(p<0,01)$

***статистически значимая разница $(p<0,001)$

Как видно из таблицы, чаще всего люди с большим количеством друзей указывают свою профессию, род деятельности или размещают рекламную информацию.

Исследователи давно отметили, что социальные сети стали популярным инструментом саморекламы (Marwick, Boyd, 2011; Quinn, Papacharissi, 2014). И полученные нами данные подтверждают это. В текущем исследовании мы заметили, что $75.2 \%$ пользователей из нашей выборки добавили в список друзей достаточно большое количество человек (более 250), поэтому можно считать, что среди них есть и незнакомцы. В связи с этим, вполне возможно, что пользователи используют данную соцсеть не только для общения, но и для рекламы. Они увеличивают размер аудитории и рассказывают в профиле о том, чем они занимаются и как с ними можно связаться для продвижения определённых товаров и услуг, поиска клиентов и возможности сотрудничать. Список друзей может стать определённой клиентской базой. Раскрытие информации о роде деятельности в профиле можно рассматривать как публичное объявление. А раскрытие контактной информации может помочь клиентам быстро связаться с владельцем профиля.

Можно заметить, что в поле «Статус» наиболее часто встречается информация рекламного характера: сведения о предлагаемых товарах или услугах, о текущем местонахождении и о возможных способах связи. По всей видимости, это наиболее удачное поле для размещения каких-либо объявлений, так как оно располагается на одной из самых заметных частей личной страницы.

Однако, как видно из таблицы, указание интересов или хобби также связано с количеством друзей, поэтому нельзя исключать и существование у пользователей социальных мотивов (например, поиск единомышленников).

Заключение (Conclusions). Подводя итог, можно отметить, что наше исследование подтвердило результаты других зарубежных работ в том, что количество сетевых друзей положительно связано с общим объемом раскрываемой в профиле информации. В связи с этим мы приходим к выводу, что данный механизм является универсальным, так как встречается в различных культурных контекстах (как у российских пользователей «ВКонтакте», так и у пользователей Facebook из других стран).

В основе данного механизма, с точки зрения многих зарубежных исследователей, лежит стремление пользователей к получению каких-либо социальных выгод (например, поиск единомышленников). Мы 
считаем данную точку зрения обоснованной, так как проведённый семантический анализ показал, что пользователи, указавшие в профиле информацию о своих увлечениях, имеют бо́льшее количество социальных связей.

В то же время в ходе нашего исследования было выявлено, что увеличение количества сетевых друзей может быть необходимо и для продвижения определенных товаров или услуг. Так, с увеличением количества связей увеличивалась частота раскрытия сведений о профессии и сфере деятельности, чаще встречались сообщения рекламного характера и контактная информация. То есть пользователи пытались одновременно и расширить аудиторию, и рассказать ей о своих услугах, то есть они преследовали рекламные мотивы. При этом наиболее частым местом для размещения рекламной информации было поле «Статус». Возможно, потому что оно расположено в наиболее заметной части личной страницы. В эпоху онлайн-маркетинга использование средств социальных сетей для рекламы и продвижения вполне естественно. И, как видим, часть людей этим пользуются.

Пытаясь дать своё объяснение парадоксу приватности, мы можем предположить следующее. Пользователи, заинтересованные в продвижении своих услуг, высоко ценят возможность бесплатной рекламы в социальной сети, и это явное преимущество значительно превышает возможный ущерб от потери приватности. Именно поэтому они раскрывают большое количество личной информации, даже если это может быть связано с определёнными рисками.

В дальнейших исследованиях необходимо изучить и другие причины раскрытия данных в профиле, а также факторы, оказывающие влияние на данный процесс. Всё это может способствовать пониманию поведения людей в социальных сетях, что также важно для разработки способов информирования пользователей о способах защиты себя и своих данных в онлайн-пространстве.

Полученные в данной работе результаты могут внести вклад в расширение теории управления приватностью Сандры Петронио, дополнив теорию новыми факторами, оказывающими влияние на самораскрытие в онлайн-пространстве.

\section{Список литературы}

Курносова Е. Социальные сети в цифрах, 2019 г. URL: https://mediascope.net/upload/iblock/f97/18.04.20 19_Mediascope_Екатерина\%20Курносова_РИФ+КИБ\%202019.pdf (дата обращения: $14.0 \overline{2} .2020)$.

Чернышов А. Г. Цифровизация и технологизация общественной жизни как социальнополитическая проблема: сохранение идентичности и роль государства в условиях развития глобальных сетей // Вестник Томского государственного университета. Философия. Социология. Политология. 2017. № 40. С. 319-328.

Сапон И. В., Леденев Д. Е. Самораскрытие пользователей в социальных сетях: теоретический обзор // Вестник НГУЭУ. 2018. № 3. C. 261-288.

Сапон И. В., Леденев Д. Е. Границы приватности пользователей социальной сети «ВКонтакте» // Научное обозрение. Серия 2. Гуманитарные науки. 2018. № 6. С. 93-105.

Aharony N. Relationships among attachment theory, social capital perspective, personality characteristics, and Facebook self-disclosure // Aslib Journal of Information Management. 2016. Vol. 68 (3). P. 362-386.

Ardi R., Maison D. How do Polish and Indonesian disclose in Facebook?: Differences in online self-disclosure, need for popularity, need to belong and self-esteem // Journal of Information, Communication and Ethics in Society. 2014. Vol. 12 (3). P. 195-218.

Bazarova N. N., Choi Y. H. Self-disclosure in social media: Extending the functional approach to disclosure motivations and characteristics on social network sites // Journal of Communication. 2014. Vol. 64 (4). P. 635-657.

Bryman A. Social research methods. Oxford: Oxford University Press, 2016.

Crabtree M. A., Pillow D. R. Extending the Dual Factor Model of Facebook Use: Social motives and network density predict Facebook use 
through impression management and open self-disclosure // Personality and Individual Differences. 2018. Vol. 133. P. 34-40.

Febriawan I. M. Convenient for Positive Expression: Role of Expressivity and Internet Use Motives to Online Self-disclosure // International Conference on Psychology and Multiculturalism. Atma Jaya Catholic University of Indonesia. Jakarta, 2017. P. 147-157.

Hollenbaugh E. E., Ferris A. L. Facebook self-disclosure: Examining the role of traits, social cohesion, and motives // Computers in Human Behavior. 2014. Vol. 30. P. 50-58.

Hollenbaugh E. E., Ferris A. L. Predictors of honesty, intent, and valence of Facebook self-disclosure // Computers in Human Behavior. 2015. Vol. 50. P. 456-464.

Kim J. Y., Chung N., Ahn K. M. Why people use social networking services in Korea: The mediating role of self-disclosure on subjective wellbeing // Information Development. 2014. Vol. 30 (3). P. 276-287.

Kim J., Lee J. E. R. The Facebook paths to happiness: Effects of the number of Facebook friends and self-presentation on subjective well-being // CyberPsychology, behavior, and social networking. 2011. Vol. 14 (6). P. 359-364.

Kisilevich S., Ang C. S., Last M. Large-scale analysis of self-disclosure patterns among online social networks users: a Russian context // Knowledge and information systems. 2012. Vol. 32 (3). P. 609-628.

Kokolakis S. Privacy attitudes and privacy behaviour: A review of current research on the privacy paradox phenomenon // Computers \& security. 2017. Vol. 64. P. 122-134.

Krasnova H., Kolesnikova E., Guenther O. «It won't happen to me!»: self-disclosure in online social networks // Proceedings of the 15th Americas Conference on Information Systems, AMCIS 2009. San Francisco, 2009. Pp. 1-10.

Lampe C. A. C., Ellison N., Steinfield C. A familiar face (book) profile elements as signals in an online social network // Proceedings of the SIGCHI conference on Human factors in computing systems. 2007. P. 435-444.

Lee E. B. Young, black, and connected: Facebook usage among African American college students // Journal of Black Studies. 2012. Vol. 43 (3). P. 336-354.

Li K., Lin Z., Wang X. An empirical analysis of users' privacy disclosure behaviors on social network sites // Information \& management. 2015. Vol. 52 (7). P. 882-891.

Marwick A. E., Boyd D. I tweet honestly, I tweet passionately: Twitter users, context collapse, and the imagined audience // New media \& society. 2011. Vol. 13 (1). P. 114-133.

Masur P. K. Theories of Self-Disclosure // Springer. 2019. P. 69-88.

Mohamed Ahmed A. A. «Sharing is Caring»: Online Self-disclosure, Offline Social Support, and Social Network Site Usage in the UAE // Contemporary Review of the Middle East. 2015. Vol. 2 (3). P. 192-219.

Quinn K., Papacharissi Z. The place where our social networks reside: Social media and sociality // Media and social life; M. B. Oliver, A. A. Raney (eds.). New York: Routledge, 2014. Pp. 159-207.

Roberts S. G. B., Dunbar R.I., Pollet T.V., Kuppens, T. Exploring variation in active network size: Constraints and ego characteristics // Social Networks. 2009. Vol. 31 (2). P. 138-146.

Schrammel J., Köffel C., Tscheligi M. Personality traits, usage patterns and information disclosure in online communities // People and Computers XXIII Celebrating People and Technology. Cambridge, 2009. P. 169-174.

Stefanone M. A., Kwon K., Lackaff D. The value of online friends: Networked resources via social network sites // First Monday. 2011. Vol. 16 (2). P. 1-17.

Stone Z., Zickler T., Darrell T. Autotagging facebook: Social network context improves photo annotation // IEEE computer society conference on computer vision and pattern recognition workshops. 2008. P. 1-8.

Trepte S., Masur P. K. Cultural differences in social media use, privacy, and self-disclosure // Research Report on a multicultural survey study. Germany: University of Hohenheim, 2016. P. 1-88.

Walther J. B. Selective self-presentation in computer-mediated communication: Hyperpersonal dimensions of technology, language, and cognition // Computers in Human Behavior. 2007. Vol. 23 (5). P. 2538-2557.

Wang Y. C., Burke M., Kraut R. Modeling self-disclosure in social networking sites // Proceedings of the 19th ACM conference on computer-supported cooperative work \& social computing. San Francisco, 2016. P. 74-85. 


\section{References}

Kurnosova, E. Social networks in numbers [Online], available at: https://mediascope.net/upload/iblock/f97/18.04.2019_Mediascope_Екатерина\%20Курносова_РИФ+КИБ\%2 02019.pdf (Accessed 14 February 2019). (in Russian).

Chernyshov, A. G. (2017), "Digitization and technologization of public life as a socio-political problem: the preservation of identity and the role of the state in the context of the development of global networks", Tomsk State University Journal. Philosophy. Sociology. Political science, (40), 319-328. (in Russian).

Sapon, I. V. and Ledenev, D. E. (2018), "Self-Disclosure on Social Network Sites: a Theoretical Review”, Bulletin NSUEM, (3), 267-288. (In Russian).

Sapon, I. V. and Ledenev, D. E. (2018), "The privacy boundaries of users of social network site VKontakte", Scientific Review. Series 2. Human Sciences, (6), 93-105. (In Russian).

Aharony, N. (2016), "Relationships among attachment theory, social capital perspective, personality characteristics, and Facebook self-disclosure", Aslib Journal of Information Management, 68 (3), 362-386.

Ardi, R. and Maison, D. (2014), "How do Polish and Indonesian disclose in Facebook?: Differences in online self-disclosure, need for popularity, need to belong and self-esteem", Journal of Information, Communication and Ethics in Society, 12 (3), 195-218.

Bazarova, N. N. and Choi, Y. H. (2014), "Self-disclosure in social media: Extending the functional approach to disclosure motivations and characteristics on social network sites", Journal of Communication, 64 (4), 635-657.

Bryman, A. (2016), Social research methods, Oxford university press, Oxford, UK.

Crabtree, M. A. and Pillow, D. R. (2018), "Extending the Dual Factor Model of Facebook Use: Social motives and network density predict Facebook use through impression management and open self-disclosure", Personality and Individual Differences, 133, 34-40.

Febriawan, I. M. (2017), "Convenient for Positive Expression: Role of Expressivity and Internet Use Motives to Online Self-disclosure", International Conference on Psychology and Multiculturalism, Atma Jaya Catholic University of Indonesia, Jakarta, Indonesia, 147-157.
Hollenbaugh, E. E. and Ferris, A. L. (2014), "Facebook self-disclosure: Examining the role of traits, social cohesion, and motives", Computers in Human Behavior, 30, 50-58.

Hollenbaugh, E. E. and Ferris, A. L. (2015), "Predictors of honesty, intent, and valence of Facebook self-disclosure", Computers in Human Behavior, 50, 456-464.

Kim, J. Y., Chung, N. and Ahn, K. M. (2014), "Why people use social networking services in Korea: The mediating role of self-disclosure on subjective well-being", Information Development, 30 (3), 276-287.

Kim, J. and Lee, J. E. R. (2011), “The Facebook paths to happiness: Effects of the number of Facebook friends and self-presentation on subjective well-being", CyberPsychology, behavior, and social networking, 14 (6), 359-364.

Kisilevich, S., Ang, C. S. and Last, M. (2012), "Large-scale analysis of self-disclosure patterns among online social networks users: a Russian context", Knowledge and information systems, 32 (3), 609-628.

Kokolakis, S. (2017), "Privacy attitudes and privacy behaviour: A review of current research on the privacy paradox phenomenon", Computers \& security, 64, 122-134.

Krasnova, H., Kolesnikova, E. and Guenther, O. (2009), "It won't happen to me!: self-disclosure in online social networks", Proceedings of the 15th Americas Conference on Information Systems, AMCIS 2009, San Francisco, USA, 1-10.

Lampe, C. A. C., Ellison, N. and Steinfield, C. (2007), "A familiar face (book) profile elements as signals in an online social network", Proceedings of the SIGCHI conference on Human factors in computing systems, 435-444.

Lee, E. B. (2012), "Young, black, and connected: Facebook usage among African American college students", Journal of Black Studies, 43 (3), 336-354.

Li, K., Lin, Z. and Wang, X. (2015), “An empirical analysis of users' privacy disclosure behaviors on social network sites", Information \& management, 52 (7), 882-891.

Marwick, A. E. and Boyd, D. (2011), "I tweet honestly, I tweet passionately: Twitter users, context collapse, and the imagined audience", New media \& society, 13 (1), 114-133.

Masur, P. K. (2019), "Theories of Self-Disclosure", Springer, 69-88.

Mohamed, A. A. A. (2015), "'Sharing is Caring': Online Self-disclosure, Offline Social 
Support, and Social Network Site Usage in the UAE", Contemporary Review of the Middle East, 2 (3), 192-219.

Quinn, K. and Papacharissi, Z. (2014), "The place where our social networks reside: Social media and sociality", in Oliver, M. B. and Raney A. A. (eds.), Media and social life, Routledge, New York, USA, 159-207.

Roberts, S. G. B., Dunbar, R. I., Pollet, T. V. and Kuppens, T. (2009), "Exploring variation in active network size: Constraints and ego characteristics", Social Networks, 31 (2), 138-146.

Schrammel, J., Köffel, C. and Tscheligi, M. (2009), "Personality traits, usage patterns and information disclosure in online communities", $\mathrm{PeO}$ ple and Computers XXIII Celebrating People and Technology, Cambridge, UK, 169-174.

Stefanone, M. A., Kwon, K. and Lackaff, D. (2011), "The value of online friends: Networked resources via social network sites", First Monday, 16 (2), 1-17.

Stone, Z., Zickler, T. and Darrell, T. (2008), "Autotagging facebook: Social network context improves photo annotation", IEEE computer society conference on computer vision and pattern recognition workshops, 1-8.

Trepte, S. and Masur, P. K. (2015), "Cultural differences in social media use, privacy, and selfdisclosure", in Research Report on a multicultural survey study, University of Hohenheim, Germany, $1-88$.

Walther, J. B. (2007), "Selective self-presentation in computer-mediated communication:
Hyperpersonal dimensions of technology, language, and cognition", Computers in Human Behavior, 23 (5), 2538-2557.

Wang, Y. C., Burke, M. and Kraut, R. (2016), "Modeling self-disclosure in social networking sites", Proceedings of the 19th ACM conference on computer-supported cooperative work \& social computing, San Francisco, USA, 74-85.

Конфликты интересов: у авторов нет конфликта интересов для декларации.

Conflicts of Interest: the authors have no conflict of interest to declare.

Сапон Ирина Валерьевна, старший преподаватель кафедры социологии, политологии и психологии Сибирского государственного университета телекоммуникаций и информатики.

Леденев Дмитрий Евгеньевич, магистрант направления подготовки «Научные исследования в области информатики и вычислительной техники» Сибирского государственного университета телекоммуникаций и информатики.

Irina V. Sapon, Senior Lecturer, Department of Sociology, Politology and Psychology, Siberian State University of Telecommunications and Informatics.

Dmitry E. Ledenev, Master's Degree Student in Computer Science and Engineering, Siberian State University of Telecommunications and Informatics. 